\title{
Association of PAI-1 rs1799889 Polymorphism with Susceptibility to Ischemic Stroke: a Huge Meta-Analysis based on 44 Studies
}

\author{
Mohammadali Jafari ${ }^{1}$, Mohammad Hossein Jarahzadeh ${ }^{2,}$, , Seyed Alireza Dastgheib ${ }^{3}$, Neda Seifi- \\ Shalamzari ${ }^{4}$, Ali Raee-Ezzabadi ${ }^{1}$, Jalal Sadeghizadeh-Yazdi ${ }^{5}$, Elahe Akbarian ${ }^{6}$, Hossein Neamatzadeh ${ }^{7,8}$
}

\begin{abstract}
Background: the PAI-1 rs1799889 polymorphism has been reported to be associated with susceptibility to ischemic stroke. However, the results of previous studies have been inconsistent or controversial. Hence, we performed a systematic review and meta-analysis to evaluate the association of PAI-1 rs1799889 polymorphism with ischemic stroke risk. Methods: A comprehensive literature search was performed on PubMed, Web of Science, Scopus, SciELO, CNKI, and CBD databases up to November 05, 2019. Pooled odds ratio (OR) with 95\% confidence interval $(\mathrm{Cl})$ were used to access the strength of this association in fixed- or random-effects model. Results: A total of 44 case-control studies with 8,620 cases and 10,260 controls were selected. Pooled data showed a significant association between PAI-1 rs1799889 polymorphism and ischemic stroke risk in the overall populations (GG vs. AA: OR $=0.791,95 \% \mathrm{Cl} 0.633-0.988, p=0.039$; GA vs. AA: $O R=0.807,95 \% \mathrm{Cl} 0.683-0.953, p=0.012 ;$ and $G G+G A$ vs. AA: OR $=0.795,95 \% \mathrm{Cl} 0.637-0.993, p=0.043$ ). Subgroup analysis by ethnicity revealed a significant association in Asian and Mixed populations, but not in Caucasians. Moreover, stratified analysis by country of origin revealed an increased risk of ischemic stroke in Chinese populations, but not among Dutch (Netherlands) and Swedish. Conclusions: This meta-analysis result suggested that PAI-1 rs1799889 polymorphism was associated with an increased risk of ischemic stroke, especially in Asian and Mixed populations.
\end{abstract}

\section{KEYWORDS}

ischemic stroke; cerebrovascular accident; PAI-1 gene; rs1799889; polymorphism; meta-analysis

\section{AUTHOR AFFILIATIONS}

${ }^{1}$ Department of Emergency Medicine, Shahid Sadoughi University of Medical Sciences, Yazd, Iran

${ }^{2}$ Department of Anesthesiology and Critical Care, Shahid Sadoughi University of Medical Sciences, Yazd, Iran

${ }^{3}$ Department of Medical Genetics, School of Medicine, Shiraz University of Medical Sciences, Shiraz, Iran

${ }^{4}$ Department of Emergency Medicine, Shahrekord University of Medical Sciences, Shahrekord, Iran

${ }^{5}$ Department of Food Science and Technology, School of Public Health, Shahid Sadoughi University of Medical Sciences, Yazd, Iran

${ }^{6}$ Children Growth Disorder Research Center, Shahid Sadoughi University of Medical Sciences, Yazd, Iran

${ }^{7}$ Department of Medical Genetics, Shahid Sadoughi University of Medical Sciences, Yazd, Iran

${ }^{8}$ Mother and Newborn Health Research Center, Shahid Sadoughi University of Medical Sciences, Yazd, Iran

* Corresponding author: Department of Anesthesiology and Critical Care, Shahid Sadoughi Hospital, Shahid Sadoughi University of Medical Sciences, Ave Sina St, Shahid Ghandi Blvd, Yazd, Iran; e-mail: drjarahzadehicm@gmail.com

Received: 12 November 2019

Accepted: 30 January 2020

Published online: 18 May 2020

Acta Medica (Hradec Králové) 2020; 63(1): 31-42

https://doi.org/10.14712/18059694.2020.13

(c) 2020 The Authors. This is an open-access article distributed under the terms of the Creative Commons Attribution License (http://creativecommons.org/licenses/by/4.0), which permits unrestricted use, distribution, and reproduction in any medium, provided the original author and source are credited. 


\section{INTRODUCTION}

Stroke is the second leading cause of death globally and leading cause of long-term disability worldwide (1). It poses a huge threat to public health and is the leading cause of death in developed and developing countries (2). It is estimated that approximately $70 \%$ of new strokes are ischemic in origin, $51 \%$ stroke death, and $58 \%$ of stroke disability-adjusted life years are because of ischemic stroke (3). The exact etiology of ischemic stroke is multifactorial and a complex interaction between modifiable and nonmodifiable conventional risk and genetic factors could be behind the pathogenesis of this disease (4). Several variants at low-penetrance and high-penetrance genes have been identified as potential ischemic stroke susceptibility loci. Numerous studies have found that Plasminogen activator inhibitor-1 (PAI-1) also serpin E1 was involved in the pathogenesis of ischemic stroke (5). Therefore, PAI-1 gene polymorphisms and its circulating levels may be associated with the development of ischemic stroke $(5,6)$.

Human PAI-1 gene is located at chromosome 7q21.3q22, contains 9 exons and spans $12.3 \mathrm{~kb}$ (7). PAI-1, a secreted single-chain glycoprotein, is one of the early inflammatory response genes, and its expression level changes dramatically in response to many stimuli, including growth factors and endotoxins $(8,9)$. Several polymorphisms within the PAI-1 gene have clearly been postulated to modulate the expression of PAI-1 $(10,11)$. Among SNPs of the PAI-1 gene, rs1799889 (4G/5G) polymorphism has been extensively studied in different disease $(7,12)$. PAI-1 rs1799889 is an inserted or deleted in the $4 \mathrm{G}$ sequence polymorphism in the PAI-1 promoter $(4 \mathrm{G} / 5 \mathrm{G})$ at $675 \mathrm{bp}$ upstream from the start of transcriptional start site in the promoter region. Studying the association of PAI-1 gene with different disease will help us to understand the mechanism of PAI- 1 regulation and the role of PAI-1 in many physiological and pathological processes $(12,13)$.

Studies have shown that the $4 \mathrm{G} / 4 \mathrm{G}$ genotype has been linked to higher PAI-1 level, compared with the $5 \mathrm{G} / 5 \mathrm{G}$ genotype, with the heterozygous genotype associated with intermediate levels. In 2003, Chen et al., have reported that PAI-1 rs1799889 polymorphism alone is not associated with an increased risk of ischemic stroke. However, they revealed a significant contribution of PAI-1 4G/4G genotype with an increased triglyceride and decreased HDL cholesterol levels in the healthy group (14). There are several numbers of epidemiological studies have evaluated association between PAI-1 rs1799889 polymorphism and ischemic stroke risk, but their results were inconsistent or even contradictory. For example, Adamski et al., and Esparza-García et al., have reported that PAI-1 rs1799889 polymorphism was not associated with an increased risk of ischemic stroke in Polish and Mexican populations, receptively $(15,16)$. By contrast, Xu et al. results supported that PAI-1 rs1799889 polymorphism might be associated with an increased risk of ischemic stroke in Han Chinese (17). In recent years, some studies already studied potential associations PAI-1 rs1799889 polymorphism with risk of ischemic stroke. Nevertheless, the results of these studies were not always consistent and the sample size of each study was also statistically insufficient. Thus, we performed a meta-analysis to offer a more comprehensive estimation of the association between PAI-1 rs1799889 and ischemic stroke susceptibility in globally populations.

\section{MATERIALS AND METHODS}

\section{SEARCH STRATEGY}

We have performed a comprehensive literature search in PubMed, MEDLINE, EMBASE, Cochrane Library, Web of Science, Elsevier, SciELO, SID, WanFang, VIP, Chinese Biomedical Database (CBD) and Chinese National Knowledge Infrastructure (CNKI) to identify all eligible studies on PAI-1 4G/5G (rs1799889) polymorphism and risk of ischemic stroke up to November 05, 2019. The following keywords were adopted in the electronic searches: ("Ischemic Strok" OR "Atherothrombotic Cerebral Infarction") AND ("Plasminogen Activator Inhibitor-1 Gene" OR "PAI-1" OR "SERPINE1") AND ("insertion/deletion polymorphism" OR " $4 \mathrm{G} / 5 \mathrm{G}$ polymorphism" OR " $4 \mathrm{G} / 5 \mathrm{G}$ promoter polymorphism” OR “rs1799889” OR “-675 4G/5G”) AND ("Gene” OR "Genotype" OR "Polymorphism SNP” OR "Mutation” OR "Variation" OR "Variant"). Publication language was restricted to English, Chinese, and Farsi. Also a manual search of the reference lists performed to retrieved articles for additional potential studies.

\section{INCLUSION AND EXCLUDING CRITERIA}

The inclusion criteria for the gene association studies in this meta-analysis were as follows: 1) studies with case-control or cohort design; 2) full-text published studies; 3) studies evaluated the association between PAI-1 rs1799889 polymorphism and ischemic stroke risk; and 4) provided the genotype distribution in both cases and controls for estimating an odds ratio (OR) with 95\% confidence interval (CI). Additionally, studies were excluded if one of the following criteria was fulfilled: 1) studies without detailed raw data regarding PAI-1 rs1799889 polymorphism; 2) case only studies; 3 ) family-based, sibling, twins and linkage studies; 4) abstracts, review, letters, comments, conference editorials, presentations, case reports, case series previous meta-analyses; 5) duplicates or overlapping studies. If the authors published two or more studies using the same data (with overlapping data), the newest publication or the publication with the largest sample size was included. There was no any limitation by ethnicity, race, placed or geography area. Moreover, non-English publications were translated and included in the meta-analysis.

\section{DATA EXTRACTION}

Two authors (HN and MJA) systematically extracted data from all eligible studies using a standardized form. Then, they have checked the data extraction results and reached consensus. If different results were generated, the two authors carried out discussions until a consensus was reached or a third author was invited to resolve the disagreement and then a final decision were made by the 


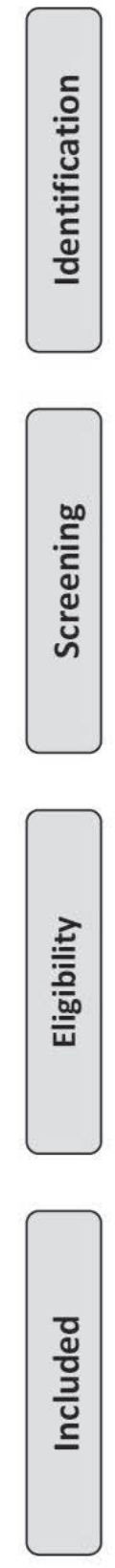

Records identified through database searching published up to November 05, 2019 $(n=289)$
Additional records identified through other sources published up to November 05, 2019 $(n=8)$

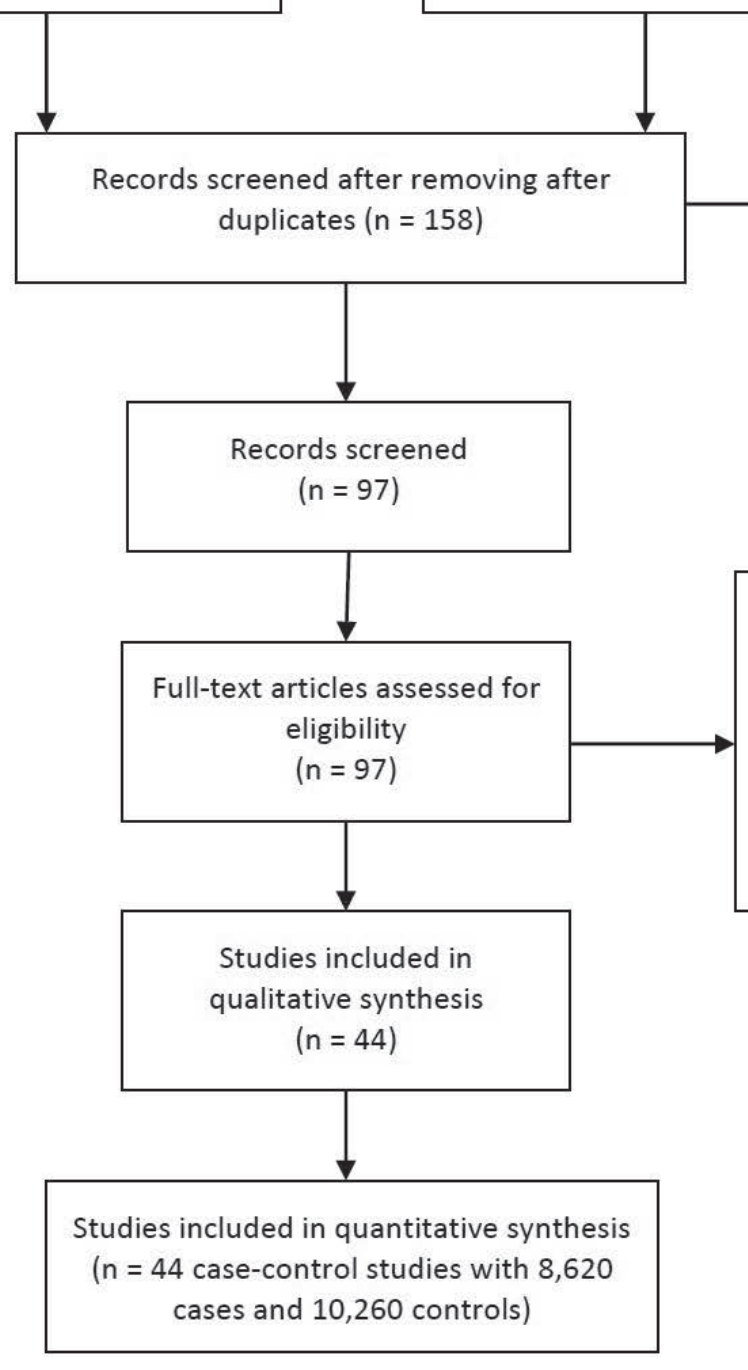

Full-text articles excluded, with reasons $(n=53$ ) Review, case reports, letters to editors, evaluated other diseases instead of Ischemic Stroke, not relevant to the PAI-1 rs1799889 SNPs.

Fig. 1 The study selection and inclusion process.

majority of the votes. The collected data were: first author's name, publication year, country of origin, ethnicity (Caucasian, Asian, African, Mixed populations), total numbers of cases and controls, genotypes frequencies of cases and controls, minor allele frequencies (MAFs) and Hardy-Weinberg equilibrium test in control subjects.

\section{STATISTICAL ANALYSIS}

An ethical approval was not necessary as this study was a meta-analysis based on previous studies. The strength of the associations PAI-1 rs1799889 (4G/5G) polymorphism and susceptibility to ischemic stroke was measured by odds ratios (ORs) with $95 \%$ confidence intervals (CIs). The statistical significance of the pooled OR was determined using the Z-test. Pooled estimates of the OR were obtained by calculating a weighted average of OR from each study. The pooled ORs was calculated under all five genetic models, i.e., allele (G vs. A), homozygote (GG vs. AA), hetero- zygote (GA vs. AA), dominant (GG+GA vs. AA) and recessive (GG vs. $G A+A A)$. Between-studies heterogeneity was assessed by a Chi-squared Q-test and $\mathrm{I}^{2}$ statistics $(\mathrm{P}<0.05)$. The heterogeneity between studies was estimated by Cochran's $\chi 2$ based Q-statistic test, in which it was considered to be statistically significant at $P \leq 0.01$. In addition, $\mathrm{I}^{2}$ test was used to quantify the effect of heterogeneity, with the range of 0 to $100 \%$, and $0-40 \%$ meant no risk of heterogeneity, 30-60\% meant a low risk of heterogeneity, $50-90 \%$ meant substantial heterogeneity and $75-100 \%$ meant considerable heterogeneity. Accordingly, when between-study heterogeneity existed a random-effects model weighted (the DerSimonian-Laird method) was applied to give a more conservative result; otherwise, a fixed-effects model weighted (the Mantel-Haenszel method) method was selected. Hardy-Weinberg equilibrium (HWE) of the genotype distribution in controls was conducted by Pearson's $\chi^{2}$ test, in which it was considered to be statistically significant at $P \leq 0.05$. A subgroup analysis by 
Tab. 1 Characteristics of studies included in this meta-analysis.

\begin{tabular}{|c|c|c|c|c|c|c|c|c|c|c|c|c|c|c|}
\hline \multirow{3}{*}{ First Author } & \multirow{3}{*}{$\begin{array}{l}\text { Country } \\
\text { (Ethnicity) }\end{array}$} & \multirow{3}{*}{$\begin{array}{l}\text { Case/ } \\
\text { Control }\end{array}$} & \multicolumn{5}{|c|}{ Cases } & \multicolumn{5}{|c|}{ Controls } & \multirow{3}{*}{ MAFs } & \multirow{3}{*}{ HWE } \\
\hline & & & \multicolumn{3}{|c|}{ Genotypes } & \multicolumn{2}{|c|}{ Allele } & \multicolumn{3}{|c|}{ Genotypes } & \multicolumn{2}{|l|}{ Allele } & & \\
\hline & & & AA & AG & GG & A & G & AA & AG & GG & A & G & & \\
\hline Catto 1997 & UK(Caucasian) & $558 / 172$ & 150 & 274 & 134 & 574 & 542 & 56 & 80 & 36 & 192 & 152 & 0.442 & 0.454 \\
\hline Liu 1998 & ina(Asian) & $107 / 95$ & 44 & 43 & 20 & 131 & 83 & 25 & 48 & 22 & 98 & 92 & 0.484 & 0.910 \\
\hline Jeppesen 1998 & Denmark(Caucasian) & $177 / 93$ & 48 & 92 & 37 & 188 & 166 & 26 & 49 & 18 & 101 & 85 & 0.457 & 0.552 \\
\hline Endler 2000 & Austria(Caucasian) & $136 / 115$ & 42 & 63 & 31 & 147 & 125 & 48 & 48 & 19 & 144 & 86 & 0.373 & 0.287 \\
\hline Elbaz 2001 & Netherlands(Caucasian) & $461 / 461$ & 125 & 223 & 113 & 473 & 449 & 129 & 245 & 87 & 503 & 419 & 0.454 & 0.123 \\
\hline Gottl 2001 & Germany(Caucasian) & $198 / 951$ & 65 & 91 & 42 & 221 & 175 & 275 & 473 & 203 & 1023 & 879 & 0.462 & 0.988 \\
\hline Bang 2001 & Korea(Asian) & $60 / 100$ & 25 & 25 & 10 & 75 & 45 & 21 & 53 & 26 & 95 & 105 & 0.525 & 0.530 \\
\hline Sun 2001 & China(Asian) & $50 / 60$ & 21 & 20 & 9 & 62 & 38 & 15 & 30 & 15 & 60 & 60 & 0.500 & 1.000 \\
\hline Zhang 2001a & China(Asian) & $95 / 60$ & 50 & 31 & 14 & 131 & 59 & 15 & 30 & 15 & 60 & 60 & 0.500 & 1.000 \\
\hline Zhang 2001b & China(Asian) & $65 / 60$ & 28 & 25 & 12 & 81 & 49 & 16 & 35 & 9 & 67 & 53 & 0.441 & 0.157 \\
\hline Kain 2002 & UK(Caucasian) & $101 / 102$ & 22 & 58 & 21 & 102 & 100 & 36 & 54 & 12 & 126 & 78 & 0.382 & 0.075 \\
\hline Hindorff 2002 & USA(Caucasian) & $41 / 385$ & 7 & 24 & 10 & 38 & 44 & 115 & 187 & 83 & 417 & 353 & 0.458 & 0.668 \\
\hline Crainich 2003 & USA(Caucasian) & $265 / 753$ & 81 & 143 & 41 & 305 & 225 & 200 & 387 & 166 & 787 & 719 & 0.477 & 0.410 \\
\hline Zhang 2003 & China(Asian) & $113 / 121$ & 48 & 47 & 18 & 143 & 83 & 23 & 70 & 28 & 116 & 126 & 0.521 & 0.080 \\
\hline Chen 2003 & Taiwan(Asian) & $100 / 150$ & 40 & 46 & 14 & 126 & 74 & 58 & 68 & 24 & 184 & 116 & 0.386 & 0.588 \\
\hline Zhan 2003 & China(Asian) & $54 / 83$ & 11 & 30 & 13 & 52 & 56 & 25 & 30 & 6 & 80 & 42 & 0.344 & 0.485 \\
\hline Guan 2004 & ina(Asian) & $222 / 215$ & 75 & 105 & 42 & 255 & 189 & 46 & 121 & 48 & 213 & 217 & 0.504 & 0.065 \\
\hline Yeh 2004 & China(Asian) & $213 / 200$ & 79 & 103 & 31 & 261 & 165 & 71 & 102 & 27 & 244 & 156 & 0.390 & 0.309 \\
\hline Yi 2004 & China(Asian) & $52 / 57$ & 20 & 22 & 10 & 62 & 42 & 28 & 27 & 2 & 83 & 31 & 0.271 & 0.138 \\
\hline Tang 2005 & China(Asian) & $122 / 50$ & 66 & 35 & 21 & 167 & 77 & 13 & 26 & 11 & 52 & 48 & 0.48 & 0.768 \\
\hline Jood 2005 & Sweden(Caucasian) & $600 / 600$ & 162 & 307 & 131 & 631 & 569 & 186 & 280 & 134 & 652 & 548 & 0.456 & 0.144 \\
\hline Van Goor 2005 & Netherlands(Caucasian) & $123 / 123$ & 33 & 61 & 29 & 127 & 119 & 36 & 58 & 29 & 130 & 116 & 0.472 & 0.550 \\
\hline Wiklund 2005a & Sweden(Caucasian) & $89 / 218$ & 42 & 33 & 14 & 117 & 61 & 67 & 109 & 42 & 243 & 193 & 0.442 & 0.844 \\
\hline Wiklund 2005b & Sweden(Caucasian) & $222 / 542$ & 94 & 85 & 43 & 273 & 171 & 174 & 261 & 107 & 609 & 475 & 0.438 & 0.609 \\
\hline Xu 2006 & China(Asian) & $72 / 77$ & 15 & 29 & 28 & 59 & 85 & 5 & 35 & 37 & 45 & 109 & 0.707 & 0.386 \\
\hline Komitopoulou & Greece(Caucasian) & $87 / 101$ & 23 & 50 & 14 & 96 & 78 & 23 & 55 & 23 & 101 & 101 & 0.500 & 0.370 \\
\hline Attia 2007 & Australia(Caucasian) & $171 / 182$ & 63 & 71 & 37 & 197 & 145 & 62 & 89 & 31 & 213 & 151 & 0.415 & 0.922 \\
\hline Saidi 2007 & Tunisia(African) & $135 / 118$ & 23 & 74 & 38 & 120 & 150 & 33 & 58 & 27 & 124 & 112 & 0.475 & 0.875 \\
\hline Liu 2008 & China(Asian) & $220 / 140$ & 48 & 114 & 58 & 210 & 230 & 43 & 70 & 27 & 156 & 124 & 0.497 & 0.876 \\
\hline Tang 2008 & China(Asian) & $90 / 30$ & 40 & 36 & 16 & 116 & 68 & 6 & 19 & 5 & 31 & 29 & 0.483 & 0.142 \\
\hline Adamski 2009 & Poland(Caucas & $390 / 291$ & 120 & 189 & 81 & 429 & 351 & 89 & 136 & 66 & 314 & 268 & 0.377 & 0.018 \\
\hline Sabino 2011 & Brazil(Mixed) & $127 / 201$ & 33 & 52 & 42 & 118 & 136 & 93 & 65 & 43 & 251 & 151 & 0.376 & $\leq 0.001$ \\
\hline Balcerzyk 2011 & Poland(Caucasian) & $70 / 133$ & 23 & 35 & 12 & 81 & 59 & 47 & 60 & 26 & 154 & 112 & 0.421 & 0.389 \\
\hline Pruissen 2011 & Netherlands(Caucasian) & $841 / 310$ & 261 & 111 & 29 & 633 & 169 & 71 & 157 & 82 & 299 & 321 & 0.518 & 0.802 \\
\hline Maguire 2011 & Australia(Caucasian) & $612 / 600$ & 198 & 279 & 135 & 675 & 549 & 169 & 302 & 129 & 640 & 560 & 0.467 & 0.784 \\
\hline Assawamakin 2 & Taiwan(Asian) & 29 & D & 7 & 31 & 199 & 159 & 67 & 110 & 52 & 244 & 14 & 467 & 0.594 \\
\hline Babu 2012 & India(Asian) & $516 / 513$ & 236 & 238 & 42 & 710 & 322 & 258 & 223 & 32 & 739 & 287 & 0.284 & 0.028 \\
\hline Huang 2014 & China(Asian) & $285 / 919$ & 115 & 156 & 14 & 386 & 184 & 310 & 520 & 89 & 1140 & 698 & 0.380 & $\leq 0.001$ \\
\hline Natesirinilkul 2014 & Thailand(Asian) & $29 / 40$ & 2 & 20 & 7 & 24 & 34 & 1 & 32 & 7 & 34 & 46 & 0.575 & $\leq 0.001$ \\
\hline Supanc 2014 & Croatia(Caucasian) & $155 / 150$ & 44 & 51 & 60 & 139 & 171 & 28 & 46 & 76 & 102 & 198 & 0.660 & $\leq 0.001$ \\
\hline García 2015 & Mexico(Mixed) & $204 / 204$ & 23 & 94 & 87 & 140 & 268 & 16 & 87 & 101 & 119 & 289 & 0.708 & 0.646 \\
\hline Ranellou 2015 & Greece(Caucasian) & $40 / 65$ & 2 & 36 & 2 & 0 & 40 & 4 & 44 & 17 & 52 & 78 & 0.600 & $\leq 0.001$ \\
\hline Akhter 2017 & India(Asian) & $100 / 100$ & 34 & 56 & 10 & 124 & 76 & 24 & 54 & 22 & 102 & 98 & 0.490 & 0.421 \\
\hline Coen Herak 2017 & Croatia(Caucasian) & $73 / 100$ & 19 & 37 & 17 & 75 & 71 & 27 & 53 & 20 & 107 & 93 & 0.465 & 0.514 \\
\hline
\end{tabular}


A

Study name

$\begin{array}{lrrrrrr} & \begin{array}{r}\text { Odds } \\ \text { ratio }\end{array} & \text { limit } & \text { Upper } & & \\ & & \text { limit } & \text { Z-Value } & \text { p-Value } \\ \text { Catto 1997 } & 1.390 & 0.861 & 2.244 & 1.346 & 0.178 \\ \text { Liu 1998 } & 0.517 & 0.237 & 1.126 & 1.661- & 0.097 \\ \text { Jeppesen 1998 } & 0.008 & 0.000 & 0.131 & 3.375- & 0.001 \\ \text { Endler 2000 } & 1.865 & 0.921 & 3.775 & 1.731 & 0.083 \\ \text { Elbaz 2001 } & 1.340 & 0.924 & 1.945 & 1.542 & 0.123 \\ \text { Gottl 2001 } & 0.875 & 0.570 & 1.343 & 0.609- & 0.542 \\ \text { Bang 2001 } & 0.323 & 0.127 & 0.820 & 2.376- & 0.017 \\ \text { Sun 2001 } & 0.429 & 0.149 & 1.236 & 1.568- & 0.117 \\ \text { Zhang 2001a } & 0.280 & 0.111 & 0.709 & 2.685- & 0.007 \\ \text { Zhang 2001b } & 0.762 & 0.264 & 2.200 & 0.503- & 0.615 \\ \text { Kain 2002 } & 2.864 & 1.181 & 6.943 & 2.328 & 0.020 \\ \text { Hindorff 2002 } & 1.979 & 0.724 & 5.414 & 1.330 & 0.184 \\ \text { Crainich 2003 } & 0.610 & 0.397 & 0.936 & 2.263- & 0.024 \\ \text { Zhang 2003 } & 0.308 & 0.142 & 0.667 & 2.985- & 0.003 \\ \text { Chen 2003 } & 0.846 & 0.391 & 1.831 & 0.425- & 0.671 \\ \text { Zhan 2003 } & 4.924 & 1.484 & 16.340 & 2.605 & 0.009 \\ \text { Guan 2004 } & 0.537 & 0.309 & 0.933 & 2.204- & 0.027 \\ \text { Yeh 2004 } & 1.032 & 0.562 & 1.894 & 0.101 & 0.919 \\ \text { Yi 2004 } & 7.000 & 1.381 & 35.478 & 2.350 & 0.019 \\ \text { Tang 2005 } & 0.376 & 0.147 & 0.964 & 2.037- & 0.042 \\ \text { Jood 2005 } & 1.122 & 0.815 & 1.546 & 0.708 & 0.479 \\ \text { Van Goor 2005 } & 1.091 & 0.542 & 2.194 & 0.244 & 0.807 \\ \text { Wiklund 2005a } & 0.532 & 0.260 & 1.090 & 1.726- & 0.084 \\ \text { Wiklund 2005b } & 0.744 & 0.482 & 1.148 & 1.337- & 0.181 \\ \text { Xu 2006 } & 0.252 & 0.082 & 0.777 & 2.400- & 0.016 \\ \text { Komitopoulou 20060.609 } & 0.252 & 1.468 & 1.105- & 0.269 \\ \text { Attia 2007 } & 1.175 & 0.650 & 2.124 & 0.533 & 0.594 \\ \text { Saidi 2007 } & 2.019 & 0.977 & 4.173 & 1.898 & 0.058 \\ \text { Liu 2008 } & 1.924 & 1.041 & 3.558 & 2.087 & 0.037 \\ \text { Tanhter 2017 } & 0.321 & 0.129 & 0.799 & 2.443- & 0.015 \\ \text { Coen Herak 2017 } & 1.208 & 0.504 & 2.892 & 0.424 & 0.672 \\ \text { Adamski 2009 } & 0.480 & 0.128 & 1.798 & 1.089- & 0.276 \\ \text { Sabing 2014 2015 } & 0.910 & 0.595 & 1.393 & 0.434- & 0.665 \\ \text { Babino 2011 } & 2.753 & 1.538 & 4.925 & 3.411 & 0.001 \\ \text { Pruissen 2011 } & 0.533 & 0.988 & 2.067- & 0.039\end{array}$

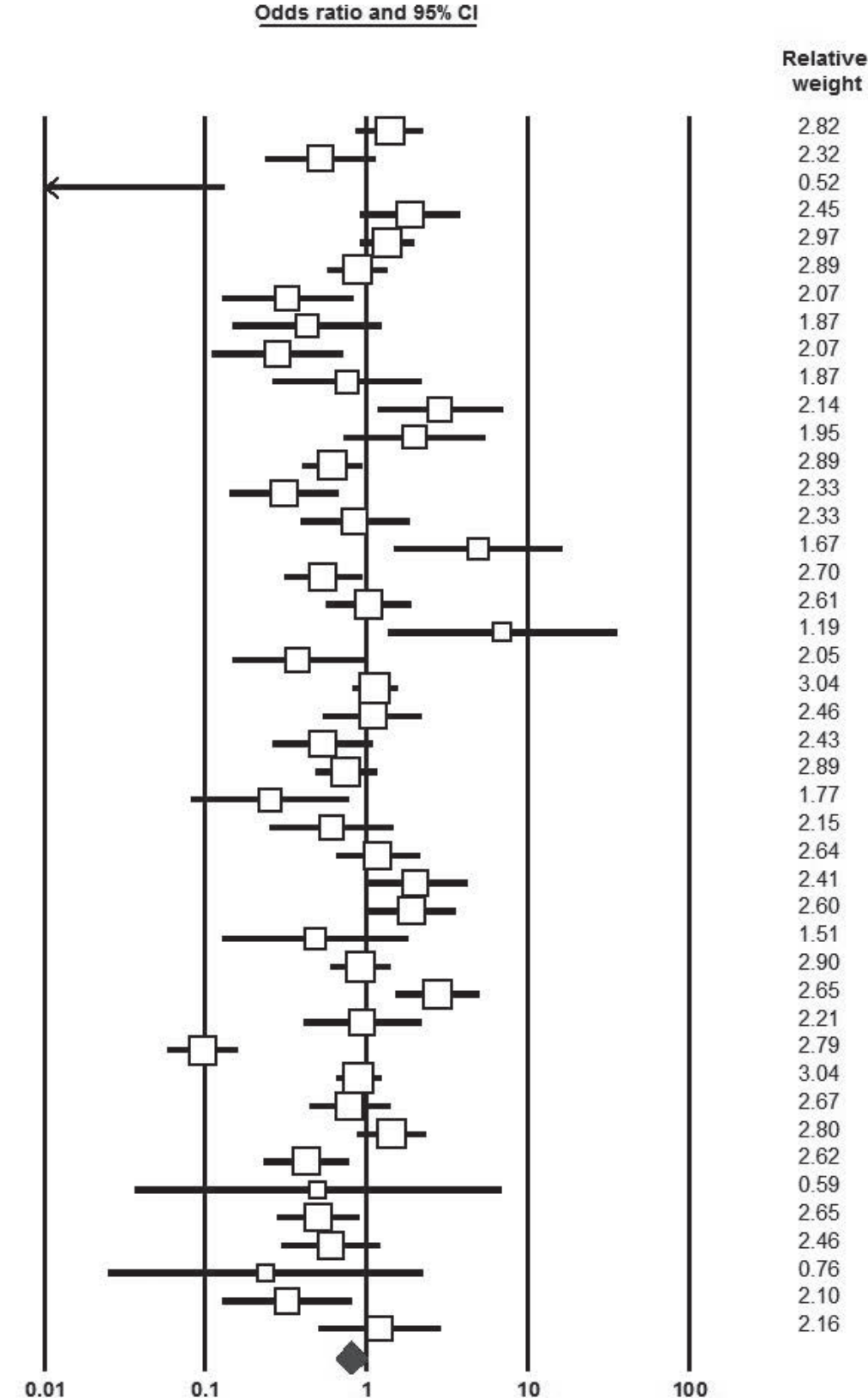

Relative

2.82

245

2.97

1.87

07

214

1.95

2.33

261

1.19

2.46

1.77

2.15

2.64

2.41
2.60

1.51

.90
65

2.21

2.79

2.67

2.80

2.62
0.59

2.65

2.46

2.10

2.16

Fig. 2A Forest plot for the association of PAI-1 rs1799889 Polymorphism with Susceptibility to Ischemic Stroke: overall population (homozygote model: GG vs. AA).

ethnicity, country of origin, and source of controls was performed to explore potential sources of between-study heterogeneity $(18,19)$. To check the stability of the pooled data, a sensitivity analysis was performed by omitting each individual study in turn from the all selected studies and reanalyzing the remainder. Moreover, sensitivity analysis was performed by excluding HWE-violating studies. The potential publication bias was explored visually by Egger's linear regression test and Begg's quantitative test (20). The asymmetric plot of Egger's test and the P-value of Begg's test less than 0.05 were considered a significant publication bias. All statistical analyses were performed using Comprehensive Meta-Analysis (CMA) Software version 2.0 (Biostat, Englewood, NJ). All tests were two-sided, and the $P$ values of $<0.05$ were considered statistically significant.

\section{RESULTS}

\section{CHARACTERISTICS OF INCLUDED STUDIES}

By electronic and manual searches concerning the association of PAI-1 rs1799889 polymorphism and ischemic stroke risk, 297 relevant studies up to November 05, 2019 were identified. After reading titles and abstracts, 139 irrelevant and duplicate articles were excluded. Another 95 articles were subsequently excluded because not reporting useful data for meta-analysis, review, case only study, and not being case-control studies. Finally, a total of 44 case-control studies $(5,14-16,21-49)$ with 8,620 ischemic stroke cases and 10,260 controls were included in the meta-analysis. Characteristics of included studies are presented in Table 1. All eligible studies were published in English and Chinese between April 1997 and November 2017. Among 
B

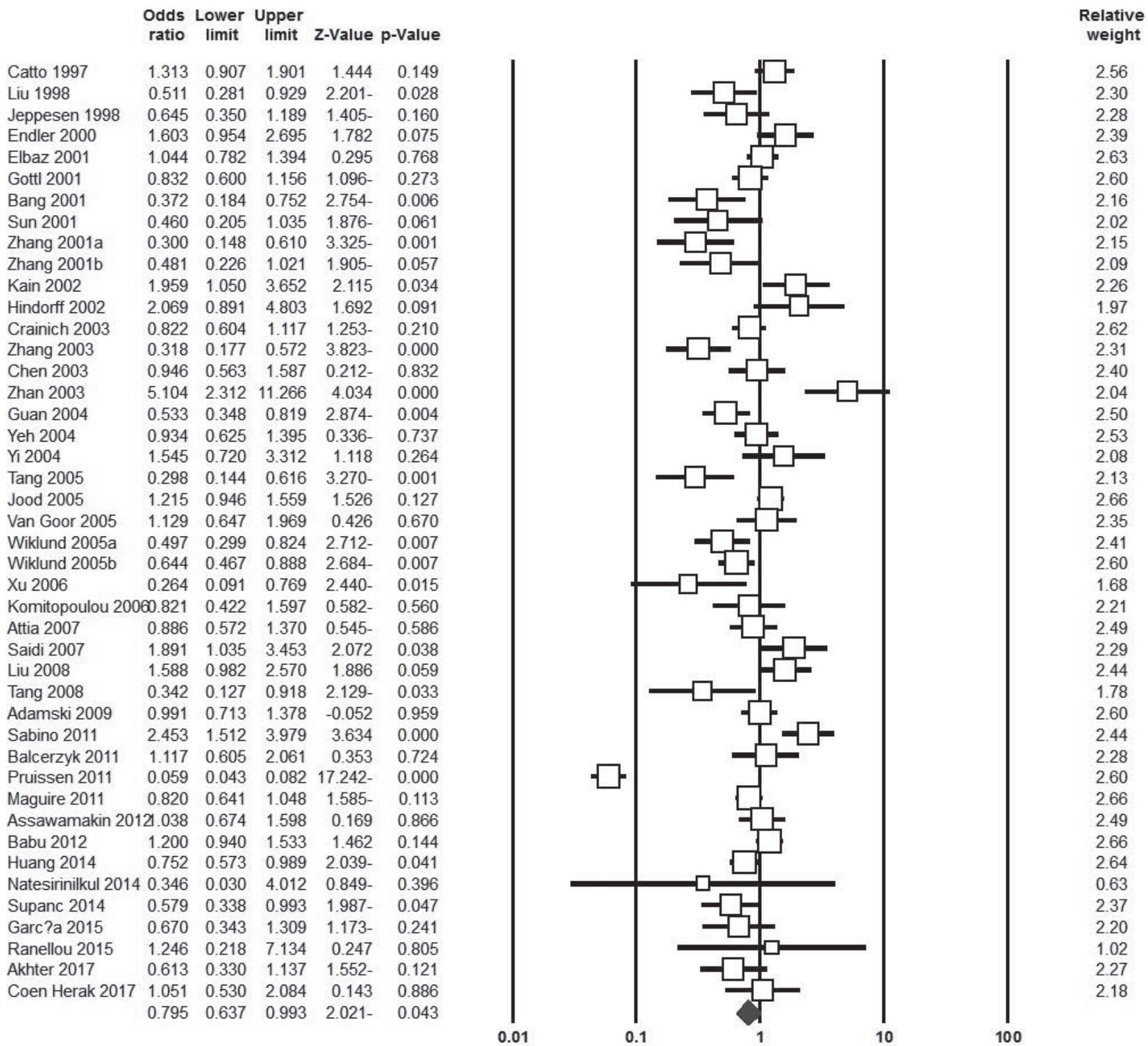

Fig. 2B Forest plot for the association of PAI-1 rs1799889 Polymorphism with Susceptibility to Ischemic Stroke: overall population (dominant model: GG + GA vs. AA).

them, 21 studies were based on Caucasian populations (5,410 cases and 6,438 controls), 20 studies based on Asian populations (3,137 cases and 3,700 controls), two studies based on mixed populations (331 cases and 405 controls), and one study was based on African populations (135 cases and 118 controls). The selected studies were conducted in UK, USA, Sweden, Greece, Australia, Austria, Poland, Denmark, Netherlands, Germany, Croatia, China, Taiwan, Thailand, Korea, India, Brazil, Mexico and Tunisia. The allele, genotype and minor allele frequency (MAF) distributions in the cases and controls are shown in Table 1. Moreover, the distribution of genotypes in the controls was in agreement with Hardy-Weinberg equilibrium (HWE) for all selected studies, except for seven studies (Table 1).

\section{QUANTITATIVE DATA SYNTHESIS}

The summary of the meta-analysis of the association of between PAI-1 rs1799889 polymorphism and ischemic stroke are shown in Table 2. Pooled data revealed that there was a significant association between PAI-1 rs1799889 polymorphism and an increased risk of ischemic stroke in the overall population under three genetic models, i.e., homozygote (GG vs. AA: OR $=0.791,95 \%$ CI $0.633-0.988$, $\mathrm{p}=0.039$, Fig 2A), heterozygote (GA vs. AA: OR $=0.807,95 \%$ CI $0.683-0.953, p=0.012)$ and dominant (GG+GA vs. AA: $\mathrm{OR}=0.795,95 \%$ CI 0.637-0.993, $\mathrm{p}=0.043$, Fig 2B). Moreover, we have performed subgroup analyses by ethnicity and country of origin. Subgroup analysis by ethnicity showed that there was a significant association between PAI-1 rs1799889 polymorphism and ischemic stroke risk in 
C

\begin{tabular}{|c|c|c|c|c|c|}
\hline \multirow[t]{2}{*}{ Study name } & \multirow[b]{2}{*}{$\begin{array}{l}\text { Odds } \\
\text { ratio }\end{array}$} & \multicolumn{3}{|c|}{ Statistics for each study } & \multirow[b]{2}{*}{ p-Value } \\
\hline & & $\begin{array}{l}\text { Lower } \\
\text { limit }\end{array}$ & $\begin{array}{l}\text { Upper } \\
\text { limit }\end{array}$ & Z-Value & \\
\hline Liu 1998 & 0.517 & 0.237 & 1.126 & $1.661-$ & \\
\hline Bang 2001 & 0.323 & 0.127 & 0.820 & $2.376-$ & \\
\hline Sun 2001 & 0.429 & 0.149 & 1.236 & $1.568-$ & \\
\hline Zhang 2001a & 0.280 & 0.111 & 0.709 & $2.685-$ & \\
\hline Zhang $2001 \mathrm{~b}$ & 0.762 & 0.264 & 2.200 & $0.503-$ & \\
\hline Zhang 2003 & 0.308 & 0.142 & 0.667 & $2.985-$ & \\
\hline Zhan 2003 & 4.924 & 1.484 & 16.340 & 2.605 & \\
\hline Guan 2004 & 0.537 & 0.309 & 0.933 & $2.204-$ & \\
\hline Yeh 2004 & 1.032 & 0.562 & 1.894 & 0.101 & \\
\hline Yi 2004 & 7.000 & 1.381 & 35.478 & 2.350 & \\
\hline Tang 2005 & 0.376 & 0.147 & 0.964 & $2.037-$ & \\
\hline Xu 2006 & 0.252 & 0.082 & 0.777 & $2.400-$ & \\
\hline Liu 2008 & 1.924 & 1.041 & 3.558 & 2.087 & \\
\hline Tang 2008 & 0.480 & 0.128 & 1.798 & $1.089-$ & \\
\hline \multirow[t]{2}{*}{ Huang 2014} & 0.424 & 0.232 & 0.775 & $2.790-$ & \\
\hline & 0.640 & 0.421 & 0.972 & 2.094- & \\
\hline
\end{tabular}

\section{Odds ratio and $95 \% \mathrm{Cl}$}

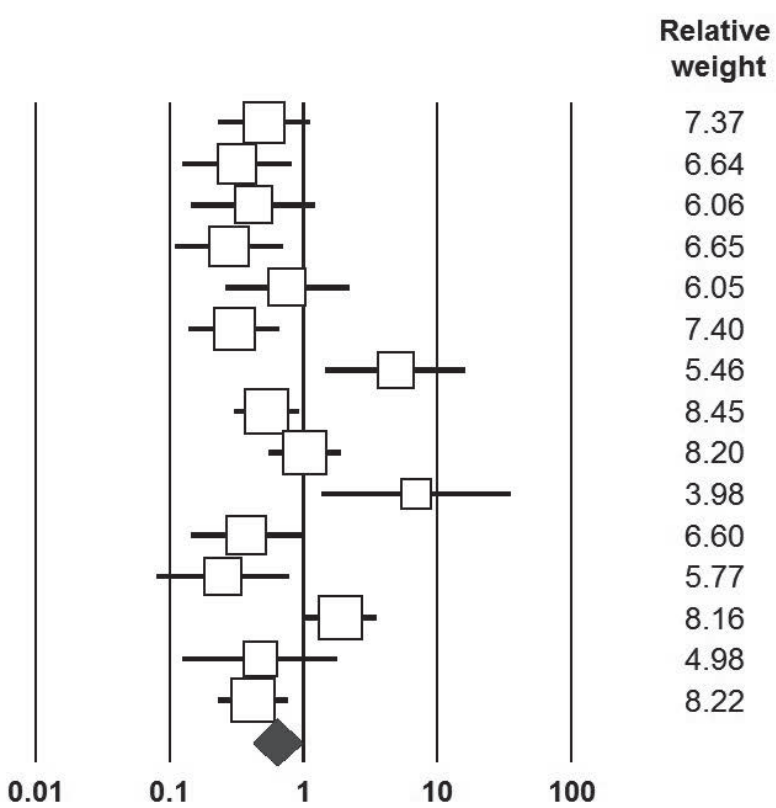

Fig. 2C Forest plot for the association of PAI-1 rs1799889 Polymorphism with Susceptibility to Ischemic Stroke: Chinese population (homozygote model: GG vs. AA).

Asians (G vs. A: OR = 0.829, 95\% CI 0.697-0.987, $\mathrm{p}=0.035$ GA vs. AA: $\mathrm{OR}=0.663,95 \%$ CI $0.518-0.848, \mathrm{p}=0.001$; and GG+GA vs. AA: OR = 0.683, 95\% CI 0.521-0.897, $\mathrm{p}=0.006$ ) and Mixed population ( $G$ vs. A: OR $=3.255,95 \% \mathrm{CI}$ 1.041-10.181, $\mathrm{p}=0.043)$, but not in Caucasians. When stratified analysis by country of origin performed a significant association was found among Chinese population (G vs. A: $\mathrm{OR}=0.798,95 \%$ CI 0.637-0.999, $\mathrm{p}=0.049 ; \mathrm{GG}$ vs. AA: $\mathrm{OR}=0.640,95 \%$ CI 0.421-0.972, $\mathrm{p}=0.036$, Fig 2C; GA vs. AA: OR = 0.577, 95\% CI 0.427-0.778, $\mathrm{p} \leq 0.001$; and GG+GA vs. $A A: O R=0.620,95 \%$ CI $0.438-0.876, p=0.007)$, but not in Dutch (Netherlands) and Swedish.

\section{BETWEEN-STUDY HETEROGENEITY TEST}

As shown in Table 2, there was statistically moderate to high between-study heterogeneity in the overall population under all five genetic models, i.e., allele $\left(\mathrm{I}^{2}=91.95, \mathrm{P}_{\mathrm{H}} \leq 0.001\right)$, homozygote $\left(\mathrm{I}^{2}=79.67, \mathrm{P}_{\mathrm{H}} \leq 0.001\right)$, heterozygote $\left(\mathrm{I}^{2}=77.98\right.$, $\left.\mathrm{P}_{\mathrm{H}} \leq 0.001\right)$, dominant $\left(\mathrm{I}^{2}=89.86, \mathrm{P}_{\mathrm{H}} \leq 0.001\right)$, and recessive $\left(\mathrm{I}^{2}=78.03, \mathrm{P}_{\mathrm{H}} \leq 0.001\right)$. To explore the potential sources of heterogeneity, subgroup analyses by ethnicity, country of origin and HWE was performed. The results suggested that the above mentioned factors did not contribute to between-study heterogeneity in the current meta-analysis.

\section{SENSITIVITY ANALYSIS}

A sensitivity analysis was used to test the effects of each study on pooled ORs. There were no significant differences observed upon removal of any of the studies, suggesting that our findings were statistically robust and reliable. Moreover, we performed sensitivity analysis by excluding the HWE-violating study (Figure 3). When this study was excluded, the results were not changed in overall population and also by subgroup analyses, indicating that our meta-analysis was statistically robust and reliable.

\section{PUBLICATION BIAS}

Begg's funnel plot and Egger's test were inspected to evaluate the possible publication bias in this meta-analysis. Results of publication bias were shown in Table 2 and Figure 4. The shape of the funnel did not show any obvious asymmetry in all of the genetic models. Moreover, Egger's test was statistically revealed that there was no a significant bias under all five genetic models in the overall populations all five genetic models, i.e., allele $\left(\mathrm{P}_{\text {Beggs }}=0.112 ; \mathrm{P}_{\text {Eggers }}=0.859\right)$, homozygote $\left(\mathrm{P}_{\text {Beggs }}=0.198 ; \mathrm{P}_{\text {Eggers }}=0.307\right)$, heterozygote $\left(\mathrm{P}_{\text {Beggs }}=0.107\right.$; $\left.\mathrm{P}_{\text {Eggers }}=0.267\right)$, dominant $\left(\mathrm{P}_{\text {Beggs }}=0.172 ; \mathrm{P}_{\text {Eggers }}=0.841\right)$, and recessive $\left(P_{\text {Beggs }}=0.723 ; P_{\text {Eggers }}=0.876\right)$.

\section{DISCUSSION}

The PAI-1 rs1799889 polymorphism association to ischemic stroke was first described by Catto et al. in 1997 (44). Since several epidemiological studies have been evaluated association between PAI-1 rs1799889 polymorphism and risk of ischemic stroke $(17,45)$. However, the results of these studies remain contradictory. It is clear that a single study may fail to demonstrate a complicated genetic relationship completely because of small sample size, which has low statistical power. Larger studies could overcome these disadvantages. Therefore, we performed a comprehensive meta-analysis of all eligible studies evaluated the association of PAI-1 rs1799889 polymorphism with risk ischemic stroke. 
Tab. 2 Summary risk estimates for association of PAI-1 rs 1799889 polymorphism with risk of ischemic stroke.

\begin{tabular}{|c|c|c|c|c|c|c|c|c|c|c|}
\hline \multirow{2}{*}{ Subgroup } & \multirow{2}{*}{ Genetic Model } & \multirow{2}{*}{ Type of Model } & \multicolumn{2}{|c|}{ Heterogeneity } & \multicolumn{4}{|c|}{ Odds Ratio } & \multicolumn{2}{|c|}{ Publication Bias } \\
\hline & & & $I^{2}(\%)$ & $P_{H}$ & OR & $95 \% \mathrm{Cl}$ & $Z_{\text {test }}$ & $P_{O R}$ & $P_{\text {Beggs }}$ & $P_{\text {Eggers }}$ \\
\hline \multirow[t]{5}{*}{ Overall } & Gvs. A & Random & 91.95 & $\leq 0.001$ & 0.854 & $0.727-1.003$ & -1.928 & 0.054 & 0.112 & 0.859 \\
\hline & GG vs. AA & Random & 79.67 & $\leq 0.001$ & 0.791 & $0.633-0.988$ & -2.067 & 0.039 & 0.198 & 0.307 \\
\hline & GA vs. $A A$ & Random & 77.98 & $\leq 0.001$ & 0.807 & $0.683-0.953$ & -2.526 & 0.012 & 0.107 & 0.267 \\
\hline & $G G+G A$ vs. $A A$ & Random & 89.86 & $\leq 0.001$ & 0.795 & $0.637-0.993$ & -2.021 & 0.043 & 0.172 & 0.841 \\
\hline & GG vs. $G A+A A$ & Random & 78.03 & $\leq 0.001$ & 0.868 & $0.726-1.038$ & -1.555 & 0.120 & 0.723 & 0.876 \\
\hline \multicolumn{11}{|l|}{ Ethnicity } \\
\hline \multirow[t]{5}{*}{ Caucasian } & G vs. A & Random & 87.76 & $\leq 0.001$ & 1.076 & $0.884-1.311$ & 0.730 & 0.465 & 0.620 & 0.561 \\
\hline & GG vs. $A A$ & Random & 56.91 & 0.003 & 1.002 & $0.807-1.243$ & 0.018 & 0.986 & 0.921 & 0.907 \\
\hline & GA vs. $A A$ & Random & 55.09 & 0.005 & 0.978 & $0.822-1.163$ & -0.255 & 0.798 & 0.373 & 0.588 \\
\hline & $G G+G A$ vs. $A A$ & Random & 61.67 & 0.001 & 0.983 & $0.825-1.172$ & -0.189 & 0.850 & 0.428 & 0.611 \\
\hline & GG vs. $G A+A A$ & Random & 48.82 & 0.017 & 0.994 & $0.839-1.178$ & -0.072 & 0.942 & 0.766 & 0.681 \\
\hline \multicolumn{11}{|l|}{ Asian } \\
\hline & G vs. A & Random & 77.84 & $\leq 0.001$ & 0.829 & $0.697-0.987$ & -2.113 & 0.035 & 0.820 & 0.389 \\
\hline & GG vs. $A A$ & Random & 94.75 & $\leq 0.001$ & 0.988 & $0.446-2.189$ & -0.031 & 0.975 & 0.581 & 0.497 \\
\hline & GA vs. AA & Random & 71.16 & $\leq 0.001$ & 0.663 & $0.518-0.848$ & -3.276 & 0.001 & 0.144 & 0.014 \\
\hline & $G G+G A$ vs. $A A$ & Random & 79.02 & $\leq 0.001$ & 0.683 & $0.521-0.897$ & -2.749 & 0.006 & 0.284 & 0.079 \\
\hline & $G G$ vs. $G A+A A$ & Random & 49.29 & 0.007 & 0.881 & $0.704-1.102$ & -1.111 & 0.267 & 0.314 & 0.410 \\
\hline \multicolumn{11}{|l|}{ Mixed } \\
\hline & G vs. A & Random & 96.28 & $\leq 0.001$ & 3.255 & $\begin{array}{c}1.041- \\
10.181\end{array}$ & 2.029 & 0.043 & NA & NA \\
\hline & GG vs. $A A$ & Random & 90.73 & 0.001 & 1.301 & $0.292-5.795$ & 0.345 & 0.730 & NA & NA \\
\hline & GA vs. $A A$ & Random & 83.11 & 0.015 & 1.333 & $0.455-3.908$ & 0.524 & 0.600 & NA & NA \\
\hline & $G G+G A$ vs. $A A$ & Random & 89.45 & 0.002 & 1.310 & $0.367-4.670$ & 0.416 & 0.678 & NA & NA \\
\hline & GG vs. $G A+A A$ & Random & 86.25 & 0.007 & 1.156 & $0.492-2.719$ & 0.333 & 0.739 & NA & NA \\
\hline \multicolumn{11}{|l|}{ Country } \\
\hline \multirow[t]{5}{*}{ China } & G vs. A & Random & 80.33 & $\leq 0.001$ & 0.798 & $0.637-0.999$ & -1.967 & 0.049 & 0.766 & 0.871 \\
\hline & GG vs. AA & Random & 71.92 & $\leq 0.001$ & 0.640 & $0.421-0.972$ & -2.094 & 0.036 & 0.373 & 0.836 \\
\hline & GA vs. AA & Random & 70.45 & $\leq 0.001$ & 0.577 & $0.427-0.778$ & -3.599 & $\leq 0.001$ & 0.373 & 0.104 \\
\hline & $G G+G A$ vs. $A A$ & Random & 80.61 & $\leq 0.001$ & 0.620 & $0.438-0.876$ & -2.706 & 0.007 & 0.766 & 0.383 \\
\hline & $G G$ vs. $G A+A A$ & Random & 52.16 & 0.010 & 0.895 & $0.680-1.178$ & -0.793 & 0.428 & 0.373 & 0.243 \\
\hline \multirow[t]{5}{*}{ Netherlands } & G vs. A & Random & 99.29 & $\leq 0.001$ & 0.498 & $0.095-2.260$ & -0.822 & 0.411 & 1.000 & 0.959 \\
\hline & GG vs. AA & Random & 96.06 & $\leq 0.001$ & 0.586 & $0.184-1.862$ & -0.907 & 0.364 & 1.000 & 0.920 \\
\hline & GA vs. AA & Random & 97.24 & $\leq 0.001$ & 0.519 & $0.089-3.014$ & -0.731 & 0.465 & 1.000 & 0.825 \\
\hline & $G G+G A$ vs. $A A$ & Random & 98.94 & $\leq 0.001$ & 0.410 & $0.052-3.208$ & -0.849 & 0.396 & 1.000 & 0.899 \\
\hline & $G G$ vs. $G A+A A$ & Random & 97.82 & $\leq 0.001$ & 0.518 & $0.093-2.885$ & -0.751 & 0.453 & 1.000 & 0.730 \\
\hline \multirow[t]{5}{*}{ Sweden } & G vs. A & Random & 75.65 & 0.016 & 0.855 & $0.647-1.129$ & -1.105 & 0.269 & 0.296 & 0.210 \\
\hline & GG vs. AA & Fixed & 56.85 & 0.099 & 0.907 & $0.711-1.155$ & -0.794 & 0.427 & 0.296 & 0.219 \\
\hline & GA vs. AA & Random & 87.54 & $\leq 0.001$ & 0.737 & $0.401-1.352$ & -0.986 & 0.324 & 1.000 & 0.341 \\
\hline & $G G+G A$ vs. $A A$ & Random & 86.60 & 0.001 & 0.751 & $0.437-1.292$ & -1.034 & 0.301 & 0.296 & 0.328 \\
\hline & $G G$ vs. $G A+A A$ & Fixed & 0.00 & 0.829 & 0.951 & $0.769-1.177$ & -0.459 & 0.646 & 0.296 & 0.321 \\
\hline \multirow[t]{5}{*}{ HWE } & G vs. A & Random & 92.62 & $\leq 0.001$ & 0.843 & $0.700-1.015$ & -1.799 & 0.072 & 0.161 & 0.964 \\
\hline & GG vs. $A A$ & Random & 80.14 & $\leq 0.001$ & 0.778 & $0.609-0.994$ & -2.010 & 0.044 & 0.277 & 0.418 \\
\hline & GA vs. AA & Random & 79.04 & $\leq 0.001$ & 0.765 & $0.633-0.926$ & -2.754 & 0.006 & 0.107 & 0.346 \\
\hline & $G G+G A$ vs. $A A$ & Random & 90.70 & $\leq 0.001$ & 0.763 & $0.590-0.988$ & -2.051 & 0.040 & 0.266 & 0.932 \\
\hline & GG vs. $G A+A A$ & Random & 79.09 & $\leq 0.001$ & 0.871 & $0.714-1.062$ & -1.365 & 0.172 & 0.743 & 0.938 \\
\hline
\end{tabular}

NA: Not Applicable. 


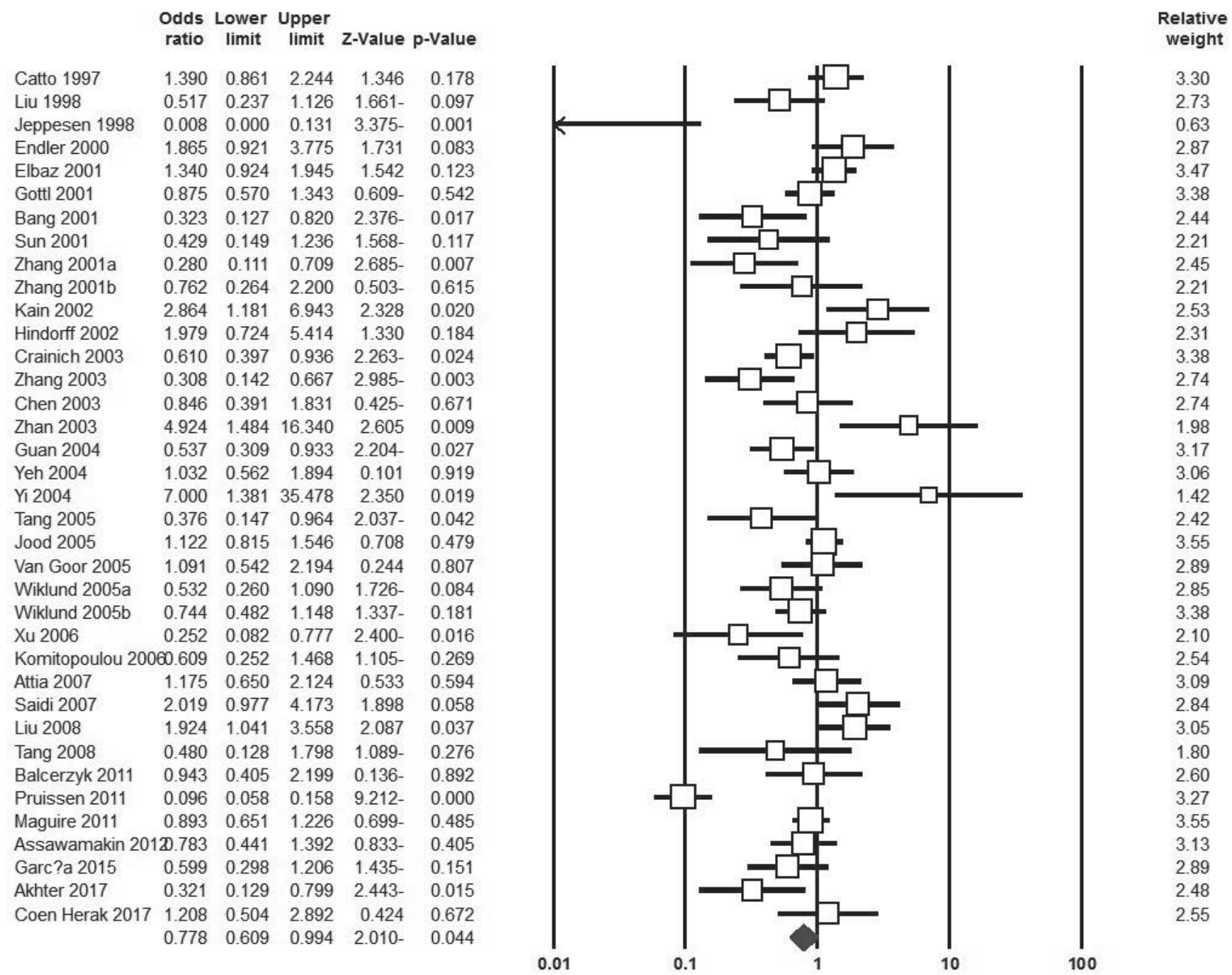

Fig. 3 Forest plot for the association of PAI-1 rs1799889 Polymorphism with Susceptibility to Ischemic Stroke after excluding HardyWeinberg equilibrium (HWE) violating studies under the homozygote genetic model (GG vs. AA).

In the current meta-analysis, we have selected a total of 44 eligible case-control studies with 8,620 ischemic stroke cases and 10,260 controls to evaluate the association of PAI-1 rs1799889 polymorphism with ischemic stroke risk. Our pooled data showed that PAI-1 rs1799889 polymorphism was significantly associated with an increased risk of ischemic stroke in the overall population. Moreover, subgroup analyses revealed that PAI-1 rs1799889 polymorphism was associated with significantly increased risk of ischemic stroke in Asian and mixed populations, but not in Caucasians. When stratified analysis by country of origin performed a significant association was found among Chinese population, but not in Dutch (Netherlands) and Swedish. This finding indicated that the carriers with the 4G allele of the PAI-1 rs1799889 polymorphism in Asians and mixed populations might be predisposed to ischemic stroke, but not in Caucasian populations. Moreover, this finding suggested a possible influence among environmental exposures and different genetic backgrounds in development of ischemic stroke in different populations. Therefore, more studies are warranted to further validate genetic background difference in the effect of PAI-1 rs1799889 polymorphism in susceptibility to ischemic stroke, especially in Caucasians. Cao et al., in a meta-analysis of eleven case-control studies with 1,358 cases and 1,134 controls evaluated the association of PAI-1 rs1799889 polymorphism with susceptibility to ischemic stroke in the Chinese population. Their results showed a significant association between PAI-1 rs1799889 polymorphism and ischemic stroke risk. However, their meta-analysis results reliability and the number of studies are considerably smaller than that needed to receive the robust conclusions (45). Here, we have extended the meta-analysis with a more relevant recently published studies and subgroup analysis by ethnicity. Moreover, Hu et al., in meta-analysis of 39 studies with 8,336 cases and 14,403 controls evaluated PAI-1 polymorphisms with risk of stroke. Their results revealed a significant association between PAI-1 rs1799889 polymorphism and an increased risk of ischemic stroke in adult, but not pediatric. Their stratified analysis showed a significant association in Asians, but not Caucasians. Moreover, they found that PAI-1-844 $\mathrm{G}>\mathrm{A}$, but not 11,053 $\mathrm{T}>\mathrm{G}$ polymorphism was associated with an increased risk of ischemic stroke and a tendency 

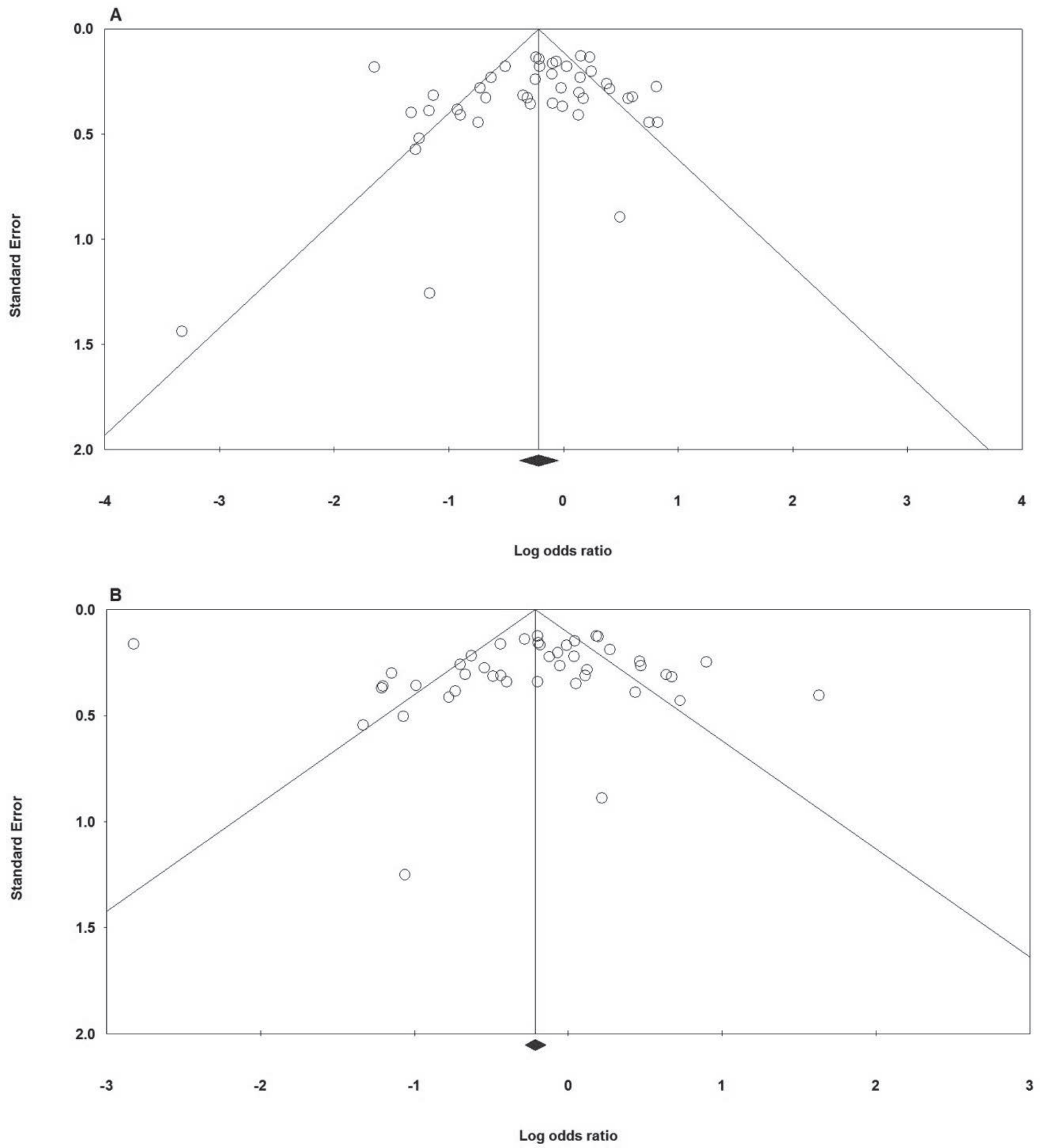

Fig. 4 Begg's funnel plots of between PAI-1 rs1799889 Polymorphism with Susceptibility to Ischemic Stroke. A: heterozygote model (GA vs. $A A)$; $B$ : dominant model (GG+GA vs. AA). Each point represents a separate study for the indicated association.

of PAI-1 rs1799889 polymorphism towards a decreased risk of hemorrhagic stroke (50).

Between-study heterogeneity is a common issue in a meta-analysis on genetic association (51-53). It could be attributable to differences in several factors such as environmental factors, including criteria or methodological factors in design and conduct of the studies $(54,55)$. Thus, identifying the potential sources of heterogeneity is one of the most important goals of meta-analysis. When all the eligible studies were pooled in this meta-analysis, there was significant between-study heterogeneity under all genetic models. However, after subgroup analyses by ethnicity the heterogeneity not effectively disappeared or decreased, which indicated that ethnicity did not play a crucial role in the existence of between-study heterogeneity in the current meta-analysis.

The current meta-analysis had some advantages. First, this was the most comprehensive and accurate me- 
ta-analysis to evaluate association of PAI-1 rs1799889 polymorphism with ischemic stroke, which involved Asian, Caucasian, mixed populations. Second, the current meta-analysis search not restricted to studies published in indexed journals. Third, we have evaluated the association under all five genetic models. Forth, there was no evidence of publication bias by Begg's funnel plot and Egger's test in this meta-analysis. Finally, sensitivity analysis confers the reliability and stability of our pooled data. However, some limitations of this meta-analysis should be mentioned. First, the sample size of the included studies was not large enough by ethnicity among African and Mixed populations. Therefore, there was a lack of statistical power to better calculate association of PAI-1 rs1799889 polymorphism with risk of stroke among African and Mixed populations. Second, all included studies were published in English or Chinese which may be brought some bias. Third, in this meta-analysis between-study heterogeneity was detected under all five genetic models in the overall population and by subgroup analyses, which may be distorting the pooled data. Finally, our results were based on single-factor estimations without adjustment for other risk factors such as age, gender, and environmental factors.

In summary, this meta-analysis result revealed that PAI-1 rs1799889 polymorphism was significantly associated with an increased risk of ischemic stroke, especially in Asian populations. Moreover, there was a significant association between PAI-1 rs1799889 polymorphism and ischemic stroke risk. Future studies with large sample sizes and well designs in the Mixed and African populations and gene-gene and gene-environment interaction studies are warranted to confirm these findings.

\section{ACKNOWLEDGEMENTS}

We are grateful to Professor Seyed Mehdi Kalantar and Professor Mohammad Hasan Sheikhha who kindly assisted the research.

\section{CONFLICT OF INTEREST}

\section{The authors declare no conflict of interest.}

\section{REFERENCES}

1. Wu J, Zhang X, Wang J, et al. Gene polymorphisms and circulating levels of the TNF-alpha are associated with ischemic stroke: A meta-analysis based on 19,873 individuals. International Immunopharmacology 2019; 75: 105827.

2. Wang L, Ge H, Peng L, Wang B. A meta-analysis of the relationship between VEGFR2 polymorphisms and atherosclerotic cardiovascular diseases. Clinical Cardiology 2019; 42(10): 860-5.

3. Feigin VL, Mensah GA, Norrving B, et al. Update on the Global Burden of Ischemic and Hemorrhagic Stroke in 1990-2013: The GBD 2013 Study Valery. Neuroepidemiology 2015; 45: 230-6.

4. Nepal G, Ojha R, Dulal HP, Yadav BK. Association between Lys198Asn polymorphism of endothelin-1 gene and ischemic stroke: A metaanalysis. Brain and Behavior 2019; 9(10): e01424.

5. Attia J, Thakkinstian A, Wang Y, et al. The PAI-1 4G/5G gene polymorphism and ischemic stroke: an association study and meta-analysis. Journal of Stroke and Cerebrovascular Diseases: The Official Journal of National Stroke Association 2007; 16: 173-9.

6. Nafría C, Fernández-Cadenas I, Mendioroz M, et al. Update on the serum biomarkers and genetic factors associated with safety and efficacy of rt-PA treatment in acute stroke patients. Stroke Research and Treatment 2011;2011: 182783

7. Kamali M, Hantoushzadeh S, Borna S, et al. Association between thrombophilic genes polymorphisms and recurrent pregnancy loss susceptibility in the iranian population: A systematic review and meta-analysis. Iranian Biomedical Journal 2018; 22 (2): 78-89.

8. Poole LG, Massey VL, Siow DL, et al. Plasminogen activator inhibitor-1 is critical in alcohol-enhanced acute lung injury in mice. American Journal of Respiratory Cell and Molecular Biology 2017; 57: 315-23.

9. Medcalf RL. Fibrinolysis, inflammation, and regulation of the plasminogen activating system. Journal of Thrombosis and Haemostasis: JTH 2007; 5(Suppl 1): 132-42.

10. Gao W, Guo Y, Bai Y, Ding X, Yan Y, Wu Z. Association between PAI-1 $4 \mathrm{G} / 5 \mathrm{G}$ polymorphism and diabetic nephropathy: a meta-analysis in the Chinese population. International Urology and Nephrology 2016; 48: 1483-9.

11. Bucan K, Plestina Borjan I, et al. Genetic Background of a Recurrent Unusual Combined Form of Retinal Vein Occlusion: A Case Report. Case Reports in Ophthalmology 2018; 9: 248-53.

12. Sobhan MR, Mahdinezhad-Yazdi M, Moghimi M, et al. Plasminogen Activator Inhibitor-1 4G/5G Polymorphism Contributes to Osteonecrosis of the Femoral Head Susceptibility: Evidence from a Systematic Review and Meta-analysis. The Archives of Bone and Joint Surgery 2018; 6: 468-77.

13. Ye Y, Vattai A, Zhang X, et al. Role of plasminogen activator inhibitor type 1 in pathologies of female reproductive diseases. International Journal of Molecular Sciences 2017;18(8): 1651.

14. Chen C-H, Eng H-L, Chang C-J, et al. 4 G/5G promoter polymorphism of plasminogen activator inhibitor-1, lipid profiles, and ischemic stroke. The Journal of Laboratory and Clinical Medicine 2003; 142: $100-5$.

15. Adamski MG, Turaj W, Slowik A, Wloch-Kopec D, Wolkow P, Szczudlik A. A-G-4G haplotype of PAI-1 gene polymorphisms -844 G/A, HindIII $G / C$, and $-6754 G / 5 G$ is associated with increased risk of ischemic stroke caused by small vessel disease. Acta Neurologica Scandinavica 2009; 120: 94-100.

16. Esparza-García JC, Santiago-Germán D, et al. GLU298ASP and 4G/5G Polymorphisms and the Risk of Ischemic Stroke in Young Individuals. The Canadian Journal of Neurological Sciences - Le Journal Canadien Des Sciences Neurologiques 2015; 42: 310-6.

17. Xu X, Li J, Sheng W, Liu L. Meta-analysis of genetic studies from journals published in China of ischemic stroke in the Han Chinese population. Cerebrovascular Diseases (Basel, Switzerland) 2008; 26: $48-62$.

18. Mousavi A, Karimi Zarchi M, Modares Gilani M, et al. Radical hysterectomy in the elderly. World Journal of Surgical Oncology 2008; 6: 38.

19. Karimi Zarchi M, Akhavan A, Gholami H, Dehghani A, Naghshi M, Mohseni F. Evaluation of cervical cancer risk-factors in women referred to Yazd-Iran hospitals from 2002 to 2009. Asian Pacific Journal of Cancer Prevention: APJCP 2010; 11: 537-8.

20. Behtash N, Karimi Zarchi M, Deldar M. Preoperative prognostic factors and effects of adjuvant therapy on outcomes of early stage cervical cancer in Iran. Asian Pacific Journal of Cancer Prevention: APJCP 2009; 10: 613-8.

21. Bang CO, Park HK, Ahn MY, Shin HK, Hwang KY, Hong SY. 4G/5G polymorphism of the plasminogen activator inhibitor-1 gene and insertion/deletion polymorphism of the tissue-type plasminogen activator gene in atherothrombotic stroke. Cerebrovascular Diseases (Basel, Switzerland) 2001;11:294-9.

22. Zhang C, Li J, Li L, Luo B. (The study of PAI-1 promotor region gene polymorphism in cerebrovascular disease). Zhonghua Yi Xue Yi Chuan Xue Za Zhi = Zhonghua Yixue Yichuanxue Zazhi = Chinese Journal of Medical Genetics 2001; 18: 383-7.

23. Kain K, Young J, Bamford J, Bavington J, Grant PJ, Catto AJ. Determinants of plasminogen activator inhibitor-1 in South Asians with ischaemic stroke. Cerebrovascular Diseases (Basel, Switzerland) 2002; 14: 77-83.

24. Hindorff LA, Schwartz SM, Siscovick DS, Psaty BM, Longstreth WT, Reiner AP. The association of PAI-1 promoter $4 \mathrm{G} / 5 \mathrm{G}$ insertion/deletion polymorphism with myocardial infarction and stroke in young women. Journal of Cardiovascular Risk 2002; 9: 131-7.

25. Crainich P, Jenny NS, Tang Z, et al. Lack of association of the plasminogen activator inhibitor-1 4G/5G promoter polymorphism with cardiovascular disease in the elderly. Journal of Thrombosis and Haemostasis: JTH 2003; 1: 1799-804.

26. Yeh P-S, Lin H-J, Li Y-H, et al. Prognosis of young ischemic stroke in Taiwan: impact of prothrombotic genetic polymorphisms. Thrombosis and Haemostasis 2004; 92: 583-9. 
27. Jood K, Ladenvall P, Tjärnlund-Wolf A, et al. Fibrinolytic gene polymorphism and ischemic stroke. Stroke 2005; 36: 2077-81.

28. Goor ML van, Gomez Garcia E, Leebeek F, et al. Blood Coagulation, Fibrinolysis and Cellular Haemostasis-The plasminogen activator inhibitor (PAI-1) 4G/5G promoter polymorphism and PAI-1 levels in ischemic stroke. A case-control study. Thrombosis and Haemostasis 2005; 93: 92-6.

29. Wiklund PG, Nilsson L, Ardnor SN, et al. Plasminogen activator inhibitor-1 4G/5G polymorphism and risk of stroke: Replicated findings in two nested case-control studies based on independent cohorts. Stroke 2005; 36: 1661-5.

30. Komitopoulou A, Platokouki H, Kapsimali Z, Pergantou H, Adamtziki E, Aronis S. Mutations and polymorphisms in genes affecting hemostasis proteins and homocysteine metabolism in children with arterial ischemic stroke. Cerebrovascular Diseases (Basel, Switzerland) 2006; 22: 13-20.

31. Saidi S, Slamia LB, Mahjoub T, Ammou SB, Almawi WY. Association of PAI-1 4G/5G and -844G/A gene polymorphism and changes in PAI-1/tPA levels in stroke: a case-control study. Journal of Stroke and Cerebrovascular Diseases: The Official Journal of National Stroke Association n.d.; 16: 153-9.

32. De Paula Sabino A, Ribeiro DD, et al. Plasminogen activator inhibitor-1 4G/5G promoter polymorphism and PAI-1 plasma levels in young patients with ischemic stroke. Molecular Biology Reports 2011; 38: 5355-60.

33. Balcerzyk A, Żak I, Emich-Widera E, et al. The plasminogen activator inhibitor-1 gene polymorphism in determining the risk of pediatric ischemic stroke--case control and family-based study. Neuropediatrics $2011 ; 42: 67-70$.

34. Pruissen DMO, Rosendaal FR, Frijns CJM, Kappelle LJ, Vos HL, Algra A. Prothrombotic gene variants and mortality after cerebral ischemia of arterial origin. Neuroepidemiology 2011; 37: 109-13.

35. Maguire J, Thakkinstian A, Levi C, et al. Impact of COX-2 rs5275 and rs20417 and GPIIIa rs5918 polymorphisms on 90-day ischemic stroke functional outcome: A novel finding. Journal of Stroke and Cerebrovascular Diseases 2011; 20: 134-44.

36. Anunchai A, Narin, Sriratanaviriyakul Yupaporn L, Wanna TOP, Tongsima S, Manop P. Meta-analysis of the plasminogen activator inhibitor-1 (PAI-1) gene with insertion/deletion $4 \mathrm{G} / 5 \mathrm{G}$ polymorphism and its susceptibility to ischemic stroke in Thai population. Asian Biomedicine 2012; 6(2): 203-17.

37. Babu MS, Prabha TS, Kaul S, et al. Association of genetic variants of fibrinolytic system with stroke and stroke subtypes. Gene 2012; 495 : 76-80.

38. Huang X, Li Y, Huang Z, Wang C, Xu Z. Pai-1 gene variants and COC use are associated with stroke risk: a case-control study in the Han Chinese women. Journal of Molecular Neuroscience: MN 2014; 54 : 803-10.

39. Natesirinilkul R, Sasanakul W, Chuansumrit A, et al. Global fibrinolytic activity, PAI-1 level, and 4G/5G polymorphism in Thai children with arterial ischemic stroke. Journal of Stroke and Cerebrovascular Diseases: The Official Journal of National Stroke Association 2014; 23: 2566-72.

40. Supanc V, Sonicki Z, Vukasovic I, Solter VV, Zavoreo I, Kes VB. The role of classic risk factors and prothrombotic factor gene mutations in ischemic stroke risk development in young and middle-aged individuals. Journal of Stroke and Cerebrovascular Diseases 2014; 23(3): e171-6

41. Ranellou K, Paraskeva A, Kyriazopoulos P, et al. Polymorphisms in prothrombotic genes in young stroke patients in Greece: a case-con- trolled study. Blood Coagulation \& Fibrinolysis: An International Journal in Haemostasis and Thrombosis 2015; 26: 430-5.

42. Akhter N, Dar SA, Chattopadhyay S, et al. Impact of p53 arg72pro SNP on Breast Cancer Risk in North Indian Population. Current Genomics 2017; 19: 395-410.

43. Coen Herak D, Lenicek Krleza J, Radic Antolic M, et al. Association of Polymorphisms in Coagulation Factor Genes and Enzymes of Homocysteine Metabolism With Arterial Ischemic Stroke in Children. Clinical and Applied Thrombosis/Hemostasis: Official Journal of the International Academy of Clinical and Applied Thrombosis/Hemostasis 2017; 23: 1042-51.

44. Catto AJ, Carter AM, Stickland M, Bamford JM, Davies JA, Grant PJ. Plasminogen activator inhibitor-1 (PAI-1) 4G/5G promoter polymorphism and levels in subjects with cerebrovascular disease. Thrombosis and Haemostasis 1997; 77: 730-4.

45. Cao Y, Chen W, Qian Y, Zeng Y, Liu W. Plasminogen activator inhibitor-1 4G/5G polymorphism and ischemic stroke risk: a meta-analysis in Chinese population. The International Journal of Neuroscience 2014; 124: 874-81.

46. Jeppesen LL, Wilhelmsen K, Nielsen LB, et al. An Insertion/Deletion polymorphism in the promoter region of the plasminogen activator inhibitor-1 gene is associated with plasma levels but not with stroke risk in the elderly. Journal of Stroke and Cerebrovascular Diseases: The Official Journal of National Stroke Association 1998; 7(6): 385-90.

47. Endler G, Lalouschek W, Exner M, Mitterbauer G, Häring D, Mannhalter $C$. The $4 \mathrm{G} / 4 \mathrm{G}$ genotype at nucleotide position -675 in the promotor region of the plasminogen activator inhibitor 1 (PAI-1) gene is less frequent in young patients with minor stroke than in controls. British Journal of Haematology 2000; 110: 469-71.

48. Elbaz A, Cambien F, Amarenco P, GENIC investigators. Plasminogen activator inhibitor genotype and brain infarction. Circulation 2001; 103: e13-4; author reply e13-4.

49. Nowak-Göttl U, Sträter R, Kosch A, et al. The plasminogen activator inhibitor (PAI)-1 promoter 4G/4G genotype is not associated with ischemic stroke in a population of German children. Childhood Stroke Study Group. European Journal of Haematology 2001; 66: 57-62.

50. Hu X, Zan X, Xie Z, et al. Association Between Plasminogen Activator Inhibitor-1 Genetic Polymorphisms and Stroke Susceptibility. Molecular Neurobiology 2017; 54: 328-41.

51. Mirjalili SA, Moghimi M, Aghili K, et al. association of promoter region polymorphisms of interleukin-10 gene with susceptibility to colorectal cancer: a systematic review and meta-analysis. Arquivos de Gastroenterologia 2018; 55: 306-13.

52. Moghimi M, Kargar S, Jafari MA, et al. Angiotensin Converting Enzyme Insertion/Deletion Polymorphism is Associated with Breast Cancer Risk: A Meta-Analysis. Asian Pac J Cancer Prev 2018; 19: 3225-31.

53. Moghimi M, Sobhan MR, Jarahzadeh MH, et al. Association of GSTM1, GSTT1, GSTM3, and GSTP1 Genes Polymorphisms with Susceptibility to Osteosarcoma: a Case- Control Study and Meta-Analysis. Asian Pac J Cancer Prev 2019; 20: 675-82.

54. Jafari-Nedooshan J, Moghimi M, Zare M, et al. Association of Promoter Region Polymorphisms of IL-10 Gene with Susceptibility to Lung Cancer: Systematic Review and Meta-Analysis. Asian Pac J Cancer Prev 2019; 20: 1951-7.

55. Farbod M, Karimi-Zarchi M, Heiranizadeh N, et al. Association of TNF- $\alpha-308$ G $>$ A Polymorphism with Susceptibility to Cervical Cancer and Breast Cancer - a Systematic Review and Meta-analysis. Klinicka Onkologie 2019; 32: 170-80. 